\title{
2.2.2 THE SIZE FREQUENCY DISTRIBUTION AND RATE OF PRODUCTION OF MICROCRATERS
}

\author{
D. A. Morrison, NASA Johnson Space Center, Houston, TX 77058 \\ E. Zinner, McDonnel1 Center for the Space Sciences, Washington University \\ St. Louis, M0 63130
}

\begin{abstract}
Crater size frequency distributions vary to a degree which probably cannot be explained by variations in lunar surface orientation of the crater detectors or changes in micrometeoroid flux. Questions of sample representativity suggest that high ratios of small to large craters of micrometeoroids (e.g., a million 1.0 micron craters for each 500 micron crater) should be the most reliable. We obtain a flux for particles producing 0.1 micron diameter craters of approximately 300 per $\mathrm{cm}^{2}$ per steradian per year. We observe no anisotropy in the submicron particle flux between the plane of the ecliptic and the normal in the direction of lunar north. No change in flux over a $10^{6}$ year period is indicated by our data.
\end{abstract}

Crater Size Frequency Distribution

Cumulative crater size frequency distributions developed on lunar rocks reflect the relative abundances of micrometeorites in terms of mass, velocity and density. Figure 1 shows size frequency distributions from various samples normalized (graphically) at a central pit diameter of 100 microns. The distribution of Fechtig et al. (1974) represents a compilation of normalized data from a large number of samples, one of which (15205) provides crater frequency data over nearly the whole range shown. We have plotted data from 15205 (Schneider et a1., 1973) separately and included counting statistics error bars to show that the variations between distributions are beyond statistical error. Data for 15015 and 15017 (Morrison et al., 1973) range from crater diameters of 0.1 to 125 microns and 0.1 to 35 microns respectively. The densities of craters from 0.1 to 2 microns and 250 to 500 microns were measured for 76215 by Morrison and Zinner (1975). Abundances of intermediate craters were not determined. Brownlee et a1. (1975) measured the distribution of craters on 60095 from 0.1 to 60 microns diameter. We have extrapolated their data to 100 microns diameter for purposes of normalization. Also included are data from 15286 for the interval 3 to 100 microns (Brownlee et al., 1973 and 1975). Data from 72315 (Hutcheon et al., 1974) are identical to 15286 over the same diameter interval and are not plotted in Figure 1 . In all cases we have plotted a distribution with a slope of -2 from a diameter of 1.0 to $\leq 0.1$ microns because a slope of -2 in this interval appears to be constant on surfaces of varying age and lunar surface orientation. The data of Hutcheon, 1975, Blanford et a1., 1974, and Morrison and Zinner (1975) show that surfaces intercepting particles with orbits normal to the plane of the ecliptic and surfaces intercepting particles confined 
to the plane of the ecliptic have the same size frequency distribution in the submicron crater diameter range. Blanford et al., 1974 and Poupeau et al., 1975 show that a -2 slope is also characteristic of submicron crater populations on surfaces exposed on or before $10^{9}$ years B.P. Both Hutcheon (1975) and Blanford et a1. (1974) have extended measurements to diameters $\leq 500 \AA$ with no change in slope indicated. A lower limit to crater diameters has not been satisfactorily established.

The discrepancies between the various size distributions cannot easily be explained. Lunar surface orientation information is lacking for all samples except 76215 for which surfaces oriented both normal and parallel to the lunar horizontal show the same size distribution, and for our sample of 15015 which had $\pi$ geometry. As we shall later discuss, the flux as well as the distribution of particles producing submicron craters does not vary sufficiently to account for the relative depletion of small craters shown, for instance, by the distribution of Fechtig et al. (1974). The exposure geometry of 76215 (Morrison and Zinner, 1975) was nearly ideal, therefore it is highly unlikely that the differences in the size distributions can be attributed to differing lunar surface orientations because an enrichment in large particle flux normal to the ecliptic would be required. A decreasing flux in the past, as postulated by Hartung and Storzer (1975), cannot account for the variations because 15205 data indicate a relative increase in large craters $(>100 \mu)$ compared to 76215 exposed for four times less, requiring an increasing flux in the past to explain the differences. Perhaps the most plausible explanation lies in the difficulty of selecting representative surfaces. On 15015 (Morrison et a1., 1973) approximately $2 \mathrm{~cm}^{2}$ were examined. Submicron and larger crater densities varied from 0 (over relatively large shielded areas) to approximately $10^{5}$ 1.0 micron craters per $\mathrm{cm}^{2}$ on the most densely cratered areas. The broad band shown in Figure 1 represents variations in crater density on the most heavily cratered surfaces. 15205 data may be biased because a relatively clean half of one fragment (Schneider et al., 1973) of a group of three from which optical data were collected was selected for SEM work. Because it was more free of secondary debris it may have had a significantly smaller solid angle and therefore not directly comparable to the other samples. It is also important to consider surface renewal by depositional processes which tend to rapidly cover small craters (Morrison et a1., 1973), and the possibility of dust coatings (Morrison and Zinner, 1975).

These arguments suggest that the distributions which show the largest ratio of small to large craters (i.e., the steepest) should be the most reliable.

\section{Micrometeoroid Flux in Space and Time}

Crater size frequency distributions can be converted to flux values if exposure ages of the surfaces can be determined. For this purpose the solar flare track production rate is the most useful clock, but it is necessary to select a solar flare energy spectrum which accurately predicts the long term track production rate. Standards for the solar flare spectrum have been proposed by Blanford et al. $(1974,1975)$, Hutcheon et a1. (1974) and Yuhas (1974). These standards differ in detail (Table 1) 
and exposure ages derived from them differ correspondingly (Morrison and Zinner, 1975). We prefer the Blanford et a1. (1975) spectrum because in this case the track production rate has been calibrated by an independent krypton isotope measurement and our track density versus depth profiles are consistent with their spectrum. The other spectra result in higher flux values.

Table 1 lists three samples which represent different situations in terms of lunar surface orientation and surface residence time and the results bear upon flux and the distribution in space of micrometeoroids. The lunar surface orientations of each are precisely known and have been described in detail by Morrison and Zinner (1975).

Submicron diameter crater densities were measured by SEM on a 11 three surfaces and the abundances of craters greater than or equal to 250 microns diameter were measured on 76215 by binocular microscope. Solar flare track density versus depth profiles were measured in each case with the plane of the track detector normal to the plane of the micrometeoroid detector. Because of the exposure geometry of the samples, we used a modification of the Yuhas (1974) track production computer program to apply corrections to the track density observed below the surface on which the crater counts were made. We emphasize that the solid angles for the crater and track detectors are the same therefore the relative flux is insensitive to errors in solid angle estimates. Data from all three surfaces for submicron craters result in the same flux of approximately 3000.1 micron diameter craters, produced per $\mathrm{cm}^{2}$ per steradian per year using the Blanford et a1. (1975) spectrum.

On 76215, densities of 250 to 500 micron diameter craters (Morrison and Zinner, 1975) and the track data indicate a production of 10-15 craters of 500 microns centra 1 pit diameter or larger produced per $\mathrm{cm}^{2}$ per $10^{6}$ years per $2 \pi$ steradians. The size frequency distribution for 15015,15017 and 76215 has been fixed to this value (10) for the production of 500 micron diameter craters (central pit), and therefore serves as a graph of crater production as indicated on the right hand ordinate of Figure 1. Table 1 also lists the track density at 100 microns depth and the abundances of 0.1 micron diameter craters for the three samples studied. The ratio of these values is useful for interlaboratory comparisons because it avoids variations in detector geometries. In all three cases the ratio we observe is one. This value is a factor of 50 larger than the value we calculate for the data of Hutcheon (1975) i.e., we observe more 0.1 micron diameter craters per track produced than Hutcheon (1975).

As is shown in Table 1, our data indicate no differences in the flux of particles between the plane of the ecliptic and the normal to the plane in the direction of lunar north. The ratio of the density of 0.1 micron diameter craters and the solar flare track density at 100 microns is approximately one in both cases, thus eliminating fortuitous differences or similarities which may have arisen from errors in solid angle measurements. Hutcheon (1975) has observed a depletion of a factor of 7 between the south ecliptic pole and the ecliptic plane over the same crater diameters.

The data in Table 1 also indicate no substantial change in flux of particles producing submicron craters over the time sampled. 76215 was exposed for approximately 
$2 \times 10^{4}$ years whereas 76015,105 and ,40 were exposed for approximately $10^{6}$ years, but the crater to track ratio and flux values are very similar, suggesting a constant flux.

The lack of anisotropy indicated by our data is surprising. Although there is inherent imprecision in our methods we believe it to be improbable that error could accumulate to more than a factor of five, and the consistent results between the three different samples of radically different exposure age and geometry shown in Table 1 tend to support this view. Mandeville (1975) has reported crater and track data for 15015. Referring the given track data to the Blanford et a1., 1975 solar flare spectrum results in an exposure age of 8-10 × 10 4 years for 15015 (Mandeville, 1975 suggests an exposure age of $2 \times 10^{3}$ years) and a crater to track ratio of 1.7 . These results are in accord with the relatively high flux values shown in Table 1 and Figure 1. Consistent results between three investigators and four samples lends credence to the size frequency distribution and crater production rates shown in Figure 1 for the curve 15015; 15017, 76215, and tend to corroborate our observation of no anisotropy. If this is the case, then useful constraints are placed upon the velocity regimes, spatial density, and physical processes acting upon submicron-sized particles at one A.U. over a long term.

\section{References}

Blanford G. E., Fruland R. M., McKay D. S., and Morrison D. A. (1974) Lunar surface phenomena: Solar flare track gradients, microcraters, and accretionary particles. Proc. Lunar Sci. Conf. 5th, pp. 2501-2526.

Blanford G. E., Fruland R. M., and Morrison D. A. (1975) Long term differential energy spectrum for solar flare iron-group particles. Proc. Lunar Sci. Conf. 6th.

Brownlee D. E., Hörz F., Vedder J. F., Gault D. E., Hartung J. B. (1973) Some physical properties of micrometeoroids. Proc. Lunar Sci. Conf. 4th, pp. 3197-3212.

Brownlee D. E., Hörz F., Hartung J. B., Gault D. E. (1975) Density chemistry and size distribution of interplanetary dust. Proc. Lunar Sci. Conf. 6th.

Fechtig H., Hartung J. B., Nagel K., and Neukum G. (1974) Lunar microcrater studies, derived meteoroid fluxes, and comparison with satellite-borne experiments. Proc. Lunar Sci. Conf. 5th, pp. 2463-2474.

Hartung J. B. and Storzer D. (1974) Lunar microcraters and their solar flare track record. Proc. Lunar Sci. Conf. 5th, pp. 2527-2541.

Hutcheon I. D., Macdouga 11 D., and Price P. B. (1974) Improved determination of the long term Fe spectrum from 1 to $460 \mathrm{MeV} / \mathrm{amu}$. Proc. Lunar Sci. Conf. 5th, pp. $2561-2576$.

Hutcheon I. D. (1975) Microcraters in oriented vugs - evidence for an anisotropy in the micrometeoroid flux. In Lunar Science VI, p. 421, The Lunar Science Institute, Houston.

Mandeville F. (1975) Microcraters observed on 15015 breccia, Abs. 6th Lunar Sci. Conf. The Lunar Science Institute, Houston.

Morrison D. A., McKay D. S., Fruland R. M., and Moore H. V. (1973) Microcraters on Apol10 15 and 16 rocks. Proc. Lunar Sci. Conf. 4th, pp. 3235-3253.

Morrison D. A., Zinner E. (1975) Studies of solar flares and impact craters in partially protected crystals. Proc. Lunar Sci. Conf. 6th.

Poupeau G., Walker R. M., Zinner E., and Morrison D. (1975) Surface exposure history of individual grains. Proc. Lunar Sci. Conf. 6th.

Schneider E., Storzer D., MehT B., Hartung J. B., Fechtig H., and Gentner W. (1973) Microcraters on Apoilo 15 and 16 samples and corresponding dust fluxes. Proc. Lunar Sci. Conf. 4th, pp. 3277-3290.

Yuhas D. E. (1974) The particle track record in lunar silicates: long-term behavior of solar and galactic VH nuclei and lunar surface dynamics. Ph.D. thesis, Washington University, St. Louis. 


\begin{tabular}{lccccc} 
Sample & $\begin{array}{c}\text { Solar Flare } \\
\text { Spectrum Used } \\
\text { for Track } \\
\text { Production }\end{array}$ & $\begin{array}{c}\text { Track Density at } \\
100 \text { Micron Depth } \\
\left(\text { Tracks } / \mathrm{cm}^{2}\right)\end{array}$ & $\begin{array}{c}\text { Pit Density } \\
(\text { Pits }>1000 \AA / \\
\left.\mathrm{cm}^{2}\right)\end{array}$ & $\begin{array}{c}\text { Exposure } \\
\text { Age } \\
(\text { Years })\end{array}$ & $\begin{array}{c}\text { Dust Particle } \\
\text { (Particles/cm } \\
\text { Steradian } / \text { Year) }\end{array}$ \\
\hline 76015,105 & $\begin{array}{c}\text { Blanford et a1. } \\
1975\end{array}$ & $6 \times 10^{7}$ & $5 \times 10^{7}$ & $4.6 \times 10^{5}$ & 311 \\
76015,40 & $1.2 \times 10^{8}$ & $10^{8}$ & $5.3 \times 10^{5}$ & 505 \\
\hline 76215 & $\begin{array}{c}\text { B1anford et a1. } \\
1975\end{array}$ & $3 \times 10^{6}$ & $5 \times 10^{6}$ & $1.6 \times 10^{4}$ & 300 \\
\hline
\end{tabular}

TABLE 1: Particle flux and data for 76015,105 (oriented parallel to ecliptic plane), 76015,40 (normal to ecliptic plane) and 76215 .

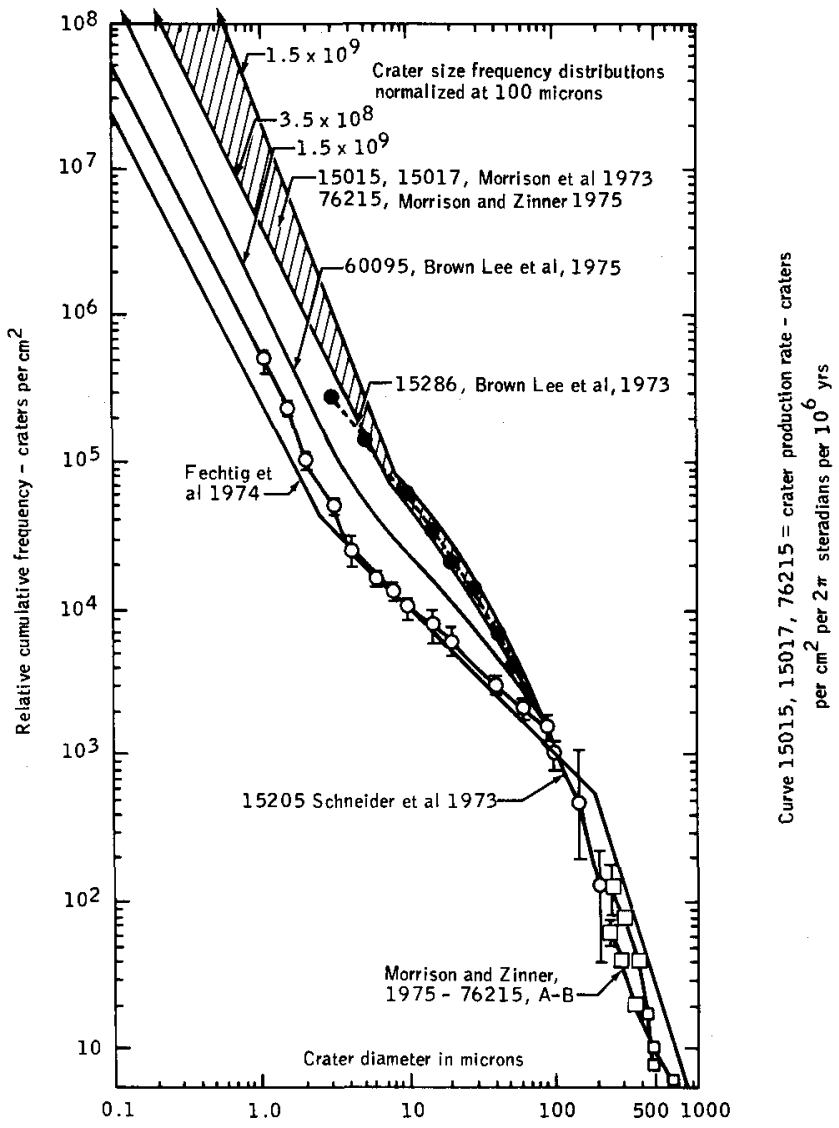

FIGURE 1: Normalized frequency distributions. Numbers for $60095 ; 15015,15017,76215$ give intercept at $0.1 \mu$. Sharp kink at $10 \mu$ in the latter is drawing artifact. 